\title{
Consumo de água pela cultura do lírio, cultivado em substratos alternativos em condições de ambiente protegido
}

\author{
Water consumption by the lily culture grown on alternative substrates in protected conditions
}

\author{
Fátima Cibele Soares ${ }^{\mathrm{I}}$ Rosmary Panno Mello ${ }^{\mathrm{II}}$ Marcia Xavier Peiter ${ }^{\mathrm{III}}$ Rogerio Antonio Belle ${ }^{\mathrm{IV}}$ \\ Adroaldo Dias Robaina ${ }^{\mathrm{III}}$ Gisele Aparecida Vivan ${ }^{\mathrm{V}}$ Ana Rita Costenaro Parizi ${ }^{\mathrm{VI}}$
}

RESUMO

O presente trabalho teve como objetivo avaliar o consumo de água em oito substratos (casca de arroz carbonizada - CAC, cinza da casca de arroz queimada - CZ, fibra de coco - FC, terra do paraíso - TP, $T P+C A C, T P+C Z$, $F C+C A C$ e $F C+C Z$ ) e definir qual desses substratos é melhor para produção comercial de lírio asiático "Orange Pixie ${ }^{\circledR ”, ~}$ cultivado em vaso e condições de ambiente protegido. $O$ experimento foi conduzido em casa de vegetação, no Departamento de Fitotecnia da UFSM, Santa Maria, RS. O delineamento experimental foi inteiramente casualizado, utilizando-se 12 repetições e três plantas por vaso para determinar a freqüência de irrigação e o consumo de água. Para as avaliações dos parâmetros, estipularam-se limites de no mínimo $60 \%$ e no máximo de $80 \%$ da capacidade de recipiente, verificado diariamente em balança digital. As determinações realizadas nos substratos foram: densidade do substrato, porosidade total, espaço de aeração e água disponível. Na planta foram determinados os seguintes parâmetros: altura da planta e da inserção da primeira flor, número de folhas e flores, distância entrenó, comprimento médio das folhas e pétalas, largura média das folhas e pétalas, consumo total de água e freqüência de irrigação. Os resultados evidenciaram que o lírio asiático "Orange Pixie ${ }^{\circledR ”}$ em vaso se adapta para cultivo em substrato de terra do paraíso e na mistura fibra de coco mais cinza de casca de arroz. Estes substratos apresentaram um consumo médio de água de 121,65 e 106,78mL e freqüência de irrigação de 22 e 21 dias, respectivamente. A melhor combinação não foi a que mais consumiu água, mas a que exigiu maior freqüência de irrigações.

Palavras-chave: irrigação, substratos, lírio asiático.

\section{ABSTRACT}

This study aimed to evaluate the consumption of water in eight substrates (rice hulls - CAC, rice husk ash burned - CZ, coconut fiber - CF, land of paradise - TP, TP +CAC, $T P+C Z, F C+C A C$ and $F C+C Z$ ) and define which of these substrates are the best for commercial production of Asiatic Lily 'Orange Pixie ${ }^{\circledR}$ “, grown in pots at protected conditions. The experiment was conducted in a greenhouse at the Department of Plant Science, UFSM, Santa Maria, RS. The experimental design was completely randomized using 12 replications and three plants per pot to determine the frequency of irrigation and water consumption. For evaluation of the parameters, it is stipulated limits of at least $60 \%$ and a maximum of $80 \%$ capacity of the container, checked daily using a digital scale. Measurements were performed on the substrates: the substrate density, total porosity, aeration and water space available. In plants, the following parameters were measured: plant height and insertion of the first flower, number of leaves and flowers, internode distance, average length of leaves and petals, average width of leaves and petals, total water consumption and irrigation frequency. The results showed that the Asiatic Lily 'Orange Pixie ${ }^{\circledR}$ "pot is suited for cultivation in the land of paradise substrate and coir mixture over rice husk ash. These substrates had an average water consumption of 121.65 and $106.78 \mathrm{~mL}$ and irrigation frequency of 22 and 21 days respectively. The best combination was not the one consumed most water, but that one that required more frequent irrigation.

Key words: irrigation, substrates, Asiatic Lily.

\footnotetext{
ICurso de Engenharia Agrícola, Universidade Federal do Pampa (Unipampa), 97546-550, Alegrete, RS, Brasil. E-mail: fatimacibele1@gmail.com. Autor para correspondência.

"Programa de Pós-graduação em Engenharia Agrícola, Universidade Federal de Santa Maria (UFSM), Santa Maria, RS, Brasil.

IIIDepartamento de Engenharia Rural, UFSM, Santa Maria, RS, Brasil.

${ }^{\text {IV }}$ Departamento de Fitotecnia, UFSM, Santa Maria, RS, Brasil.

vInstituto Federal Farroupilha (IFFarroupilha), Alegrete, RS, Brasil.

VIInstituto Sul Rio-Grandense (IFSUL), Venâncio Aires, RS, Brasil.
} 


\section{INTRODUÇÃO}

O lírio, pertencente ao gênero Lilium, família Liliaceae, é uma planta muito apreciada pela beleza de suas flores, sendo comercializado em vaso ou como flores de corte, as quais tradicionalmente são muito utilizadas na decoração de ambientes.

Os substratos, principalmente em espécies cultivadas em vaso, assumem grande importância, pois oferecem suporte ao sistema radicular, o qual dispõe de um volume de substrato limitado para o armazenamento de água e aeração, ou seja, pode limitar o desenvolvimento da planta como um todo (COUTINHO et al., 2006). Vários estudos estão sendo realizados objetivando testar e desenvolver materiais alternativos para formulação de substratos (JASMIM et al., 2006; RODRIGUES et al. 2004; SILVEIRA et al., 2002). Para SANTOS (2006) e PELIZER et al. (2007), essas iniciativas são interessantes, pois a utilização de resíduos agroindustriais disponíveis regionalmente, como componente para substratos, pode propiciar a redução de custos, assim como auxiliar na diminuição da poluição ambiental, decorrente da deposição desses materiais em locais impróprios.

BAUMGARTEN (2002) comenta que na Europa existe a preocupação em se encontrar novos substratos a fim de substituir a utilização da turfa, pois é um recurso natural não renovável. É importante observar também que é necessário desenvolver substratos de baixo custo, fácil utilização, longa durabilidade e recicláveis, ou ainda, desenvolver métodos para reaproveitá-los no cultivo convencional e melhoria das condições químicas e físicas do solo.

Para a produção de flores em vaso, além do substrato, também se faz necessário um conhecimento mais técnico para proceder às irrigações, visto que devido à regionalização e as características das culturas, é comum a utilização de substratos possuindo diferentes propriedades químicas, físicas e biológicas (BELLÉ, 2010).

A irrigação é uma prática fundamental para o cultivo de lírio, em vaso, em condição de ambiente protegido. Porém, o manejo inadequado pode resultar em prejuízos no crescimento das plantas e queda na produtividade e qualidade do produto final (FERMINO \& BELLÉ, 2008). De forma geral, o manejo da irrigação na produção de flores tem se caracterizado pelo empirismo, devido ao pouco conhecimento técnico que se tem na área, em comparação a outras culturas irrigadas tradicionalmente com maior interesse comercial. KAMPF (2000), destacou que as informações técnicas disponíveis para a produção de plantas ornamentais baseiam-se principalmente em: espaçamento, adubação, combate a pragas e doenças, ficando assim, uma lacuna em pesquisas direcionadas ao manejo da irrigação.

Geralmente, os produtores têm dificuldade para fazer o manejo racional da irrigação em flores, principalmente naquelas conduzidas em ambiente protegido, por apresentarem condições ambientais próprias, impedindo o uso direto dos métodos já consagrados para a determinação da evapotranspiração (BELLÉ, 2010). FARIAS (2006) comenta que o consumo de água pelas espécies ornamentais cultivadas em estufas é menor, devido à atenuação que ocorre na densidade de fluxo da radiação solar incidente e menor velocidade de renovação do ar junto às plantas. Pesquisas realizadas em outros países evidenciam que o consumo hídrico de espécies cultivadas em ambientes protegidos é 20 a $40 \%$ inferior, em relação ao cultivo a céu aberto (PEREIRA et al., 2005).

No Estado do Rio Grande do Sul, a pesquisa com plantas ornamentais ainda é incipiente, tanto em aspectos relacionados ao consumo hídrico como na condução do cultivo. Ressalta-se, ainda, que mesmo cultivado em pequenas áreas, o consumo hídrico das espécies ornamentais pode contribuir, quando superdimensionado no manejo, para reduzir ainda mais as nossas escassas fontes hídricas. O presente trabalho teve como objetivo avaliar o consumo de água em diferentes substratos e definir qual desses substratos é melhor para produção comercial de lírio asiático “Orange Pixie ${ }^{\circledR}$, cultivado em vaso e condições de ambiente protegido.

\section{MATERIAL E MÉTODOS}

O experimento foi conduzido no Setor de Floricultura da Universidade Federal de Santa Maria, Rio Grande do Sul, em casa de vegetação, contendo quatro bancadas de concreto, distribuídas na direção norte-sul, com dimensões de $1 \mathrm{~m}$ de largura por 2,5m de comprimento e 0.8 de altura. O local se encontra a 2941'25" de latitude, 5348'42" de longitude e $95 \mathrm{~m}$ de altitude, possuindo clima $\mathrm{Cfa}$, conforme classificação de Köppen (MORENO, 1961). A cultivar de lírio-testada foi “Orange Pixie ${ }^{\circledR ”, ~ p o i s ~ a t u a l m e n t e ~ e ́ ~ a ~ m a i s ~ u s a d a ~ n a ~}$ produção de flores de lírio em vaso.

O experimento foi conduzido em delineamento experimental inteiramente casualizado, com oito tratamentos, doze repetições e três plantas por vaso. Os vasos foram preenchidos com volume corresponde a 1,3 litros. O trabalho foi realizado com a predominância de um grupo de materiais e misturas (casca de arroz carbonizada, cinza de casca de arroz 
carbonizada e fibra de coco), considerando a disponibilidade regional e comercial, baixo custo de aquisição, possibilidade de reaproveitamento a fim de contribuir com o equilíbrio ecológico do meio ambiente.

As misturas foram realizadas manualmente, com os componentes levemente úmidos (10\% de sua capacidade de vaso) para facilitar o manuseio e a homogeneização dos mesmos. Para a proporção das misturas usou-se o critério volume por volume, conforme metodologia proposta por KAMPF, (2000).

As seguintes proporções constituíram os tratamentos analisados: Tratamento 1 (CAC) - 100\% de casca de arroz carbonizada; Tratamento 2 (CZ) $100 \%$ de cinza de casca de arroz; Tratamento 3 (FC) $100 \%$ de fibra de coco; Tratamento 4 (TP) - 100\% de terra do paraíso; Tratamento 5 (TP+CAC) - 50\% de terra do paraíso $+50 \%$ casca de arroz carbonizada; Tratamento $6(\mathrm{TP}+\mathrm{CZ})-50 \%$ de terra do paraíso $+50 \%$ de cinza de casca de arroz; Tratamento 7 (FC+CAC) $50 \%$ de fibra de coco $+50 \%$ de casca de arroz carbonizada; Tratamento 8 (FC+CZ) - 50\% de fibra de coco $+50 \%$ de cinza de casca de arroz carbonizada. Foi realizada a caracterização física dos diferentes substratos pelas análises: densidade do substrato (DS), relação poros/sólidos (P/S), espaço de aeração (EA) e retenção de água (CRA) As quantificações foram desenvolvidas de acordo com metodologia proposta por KÄMPF (2006).

A umidade dos vasos foi controlada diariamente, por pesagem individual de todos os vasos de cada tratamento. $\mathrm{O}$ volume de água foi determinado fazendo a diferença entre os limites mínimo, $\mathrm{Pv}_{\min }(60 \%)$, e máximo, $\mathrm{PV}_{\text {máx }}(100 \%)$, do peso de cada vaso, nos diferentes substratos, utilizando-se as seguintes expressões:

$\left(\mathrm{PV}_{\text {máx }}-\mathrm{PV}_{\min }\right)=\left(\mathrm{PV}_{100 \%}-\mathrm{PV}\right) .0 .2$

Em que,

$\mathrm{PV}_{\text {máx }}=\left(P V_{100 \%}-\mathrm{PV}\right) \cdot 0.8+\mathrm{PV}_{\mathrm{s}}$

$\mathrm{PV}_{\text {mín }}=\left(\mathrm{PV}_{100 \%}-\mathrm{PV}_{\mathrm{s}}^{\mathrm{s}}\right) \cdot 0.6+\mathrm{PV}_{\mathrm{s}}$

Sendo: $\mathrm{PV}_{100 \%}$ - peso de todo vaso à $100 \%$ da capacidade de retenção de água; $\mathrm{PV}_{\mathrm{S}}$ - peso do recipiente com três bulbos e o substrato seco.

No final do ciclo da cultura foram medidos: altura da planta e da inserção da primeira flor, número de folhas e flores, distância dos entrenós, comprimento médio das folhas e pétalas, e largura média das folhas e pétalas. Finalmente, realizou-se análise de variância e posterior comparação das médias pelo teste de Tukey em 5\% de probabilidade de erro, através do software ASSISTAT, (versão 7. 6, 2011)

\section{RESULTADOS E DISCUSSÃO}

Na tabela 1 encontram-se apresentados os volumes total e médio de água aplicado nas irrigações, e o número total das irrigações para os diferentes substratos testados ao longo do ciclo de cultivo (67 dias) do lírio asiático (“Orange Pixie ${ }^{\circledR ”}$ ”. Verificou-se para o volume total de água aplicado que houve diferença estatística para a maioria dos substratos testados. O substrato $\mathrm{FC}+\mathrm{CZ}$ proporcionou o maior consumo de água $(2676,33 \mathrm{~mL})$ e o substrato FC o de menor consumo (1526,33mL). O volume médio aplicado por irrigação também diferiu estatisticamente para a maioria dos substratos, sendo que o tratamento $\mathrm{FC}+\mathrm{CZ}$ exigiu maior volume médio $(121,65 \mathrm{~mL})$, entre os limites

Tabela 1 - Volume total de água aplicado no ciclo da cultura do lírio, volume médio de água aplicado por irrigação e número total de irrigações para os diferentes substratos. UFSM, Santa Maria, 2004.

\begin{tabular}{lccc}
\hline Tratamentos & Volume total de água $(\mathrm{mL})$ & Volume médio de água $(\mathrm{mL})$ & Número total de irrigações \\
\hline FC+CZ & $2.676,33 \mathrm{a}$ & $121,65 \mathrm{a}$ & 22 \\
CZ & $2.242,54 \mathrm{bc}$ & $106,78 \mathrm{~b}$ & 21 \\
TP & $2.449,61 \mathrm{ab}$ & $106,50 \mathrm{~b}$ & 23 \\
TP+CZ & $2.042,77 \mathrm{cde}$ & $92,85 \mathrm{c}$ & 22 \\
FC+CAC & $2.049,77 \mathrm{~cd}$ & $85,40 \mathrm{~cd}$ & 24 \\
FC & $1.526,33 \mathrm{f}$ & $84,79 \mathrm{~cd}$ & 18 \\
CAC & $1.904,37 \mathrm{de}$ & $76,17 \mathrm{de}$ & 25 \\
TP+CAC & $1.795,34 \mathrm{e}$ & $71,81 \mathrm{e}$ & 25 \\
Média & $2.085,89$ & 93,25 & \\
CV $(\%)$ & 9,38 & 9,54 & \\
\hline
\end{tabular}

*CAC: $100 \%$ volume de casca de arroz carbonizada; CZ: 100\% volume de cinza de casca de arroz; FC: 100\% volume de fibra de coco; TP 100\% de volume de terra do paraíso; TP+CAC: $50 \%$ volume de terra do paraíso + 50\% volume casca de arroz carbonizada; TP+CZ: $50 \%$ volume de terra do paraíso + 50\% volume de cinza de casca de arroz: FC+CAC: $50 \%$ volume de fibra de coco + 50\% volume de casca de arroz carboniza: FC+CZ: 50\% volume de fibra de coco + 50\% volume de cinza de casca de arroz carbonizada. 
mínimo e máximo estipulados, em 22 irrigações. Os substratos CAC e TP+CAC exigiram o menor volume médio (76,17 e 71,81mL, respectivamente), mas com maior número de irrigações (25 irrigações).

Analisando os resultados de Ds apresentados na tabela 2, verificou-se que somente a mistura TP apresentou Ds suficiente para garantir a estabilidade do recipiente. Os materiais CAC, CZ, FC e as misturas $\mathrm{FC}+\mathrm{CAC}$ e FC+CZ apresentaram densidade excessivamente baixas, o que comprometeu a estabilidade do vaso sob condições de aeração da estufa ou de manipulação. GAULAND (1997) comenta que densidades inferiores a $400 \mathrm{~kg} \mathrm{~m}^{-3}$ podem facilitar o tombamento de recipientes como vasos ou sacos plásticos, conferir pouco contato entre semente ou raiz da planta e o meio, dificultando a fixação e desenvolvimento do sistema radicular das plantas. O autor considera, por outro lado, que densidades muito elevadas podem dificultar a penetração e desenvolvimento do sistema radicular das plantas, além de apresentar redução no espaço poroso total e volume de poros ocupados por ar.

VERDONK \& GABRIELS (1988) consideram como ideal um valor de $\mathrm{Pt}=0,85 \mathrm{~m}^{3} \mathrm{~m}^{-3}$ para os substratos hortículas. Seguindo esta consideração, observou-se na tabela 2 que os substratos que mais se aproximaram do ideal foram as misturas TP+CAC e TP+CZ. Os demais componentes ou misturas apresentaram valores muito porosos $\left(0,90\right.$ a $\left.0,94 \mathrm{~m}^{3} \mathrm{~m}^{-3}\right)$, especialmente os materiais CAC, $\mathrm{CZ}$ e FC e, as misturas em que fizeram parte.

Os tratamentos FC, $\mathrm{TP}+\mathrm{CZ}$ e $\mathrm{FC}+\mathrm{CZ}$ foram os que obtiveram valores de EA mais próximos do ideal, ou seja, $0,30 \mathrm{~m}^{3} \mathrm{~m}^{-3}$, segundo PENNINGSFELD (1983).
O substrato contendo somente CAC foi o tratamento que apresentou maior EA. Os resultados obtidos concordam com SCHMITZ et al. (2002), que qualificaram a CAC como um ótimo material para condicionamento de substratos deficientes em aeração. Os autores observam, no entanto, que valores elevados de EA podem proporcionar deficiência hídrica às plantas, especialmente no caso de irrigações pouco frequentes.

$A$ adição de CAC à FC aumentou excessivamente o EA, enquanto que sua adição à TP não proporcional EA próxima do ideal $\left(0,30 \mathrm{~m}^{3} \mathrm{~m}^{-3}\right)$.

Segundo DE BOODT \& VERDONCK(1972) a faixa ideal de AD para plantas cultivadas em vaso deve ficar entre 0,24 a $0,40 \mathrm{~m}^{3} \mathrm{~m}^{-3}$. Desse modo, observou-se na tabela 2 que somente a CAC e a mistura $\mathrm{FC}+\mathrm{CAC}$ foram os tratamentos que apresentaram valores abaixo dos limites ideais $\left(0,14 \mathrm{e} 0,21 \mathrm{~m}^{3} \mathrm{~m}^{-3}\right.$, respectivamente). A mistura da CAC à FC proporcionou importante redução de disponibilidade de água da FC $\left(0,39\right.$ para $\left.0,21 \mathrm{~m}^{3} \mathrm{~m}^{-3}\right)$ quando em mistura. Os demais materiais e misturas não apresentaram restrições quanto à disponibilidade hídrica.

Baseando-se nos limites ideais dos atributos físico-hídricos, observou-se que todos os tratamentos testados apresentam no mínimo dois fatores limitantes. Considerando-se a densidade como atributo de baixa relevância, observou-se que a TP+CZ foi o tratamento que proporcionou as melhores características para Pt, EA e AD. Enquanto que o pior material foi o CAC, em que todas as características extrapolaram os limites ideais.

Na tabela 3, observou-se que a altura de planta apresentou amplitude de variação média de

Tabela 2 - Densidade do substrato seco (Ds), porosidade total (PT), espaço de aeração (EA) e água disponível (AD), dos materiais e das misturas formuladas com a finalidade de uso como substrato, para a produção de lírios em vaso. UFSM, Santa Maria, 2004.

\begin{tabular}{|c|c|c|c|c|}
\hline Tratamentos* & Ds $\left(\mathrm{kg} \mathrm{m}^{-3}\right)$ & $\mathrm{PT}\left(\mathrm{m}^{3} \mathrm{~m}^{-3}\right)$ & $\mathrm{EA}\left(\mathrm{m}^{3} \mathrm{~m}^{-3}\right)$ & $\mathrm{AD}\left(\mathrm{m}^{3} \mathrm{~m}^{-3}\right)$ \\
\hline CAC & 129,62 & 0,90 & 0,67 & 0,14 \\
\hline $\mathrm{CZ}$ & 157,15 & 0,91 & 0,47 & 0,33 \\
\hline FC & 91,13 & 0,94 & 0,29 & 0,39 \\
\hline $\mathrm{TP}$ & 503,88 & 0,75 & 0,19 & 0,30 \\
\hline $\mathrm{TP}+\mathrm{CAC}$ & 352,52 & 0,79 & 0,22 & 0,29 \\
\hline $\mathrm{TP}+\mathrm{CZ}$ & 334,08 & 0,83 & 0,34 & 0,31 \\
\hline $\mathrm{FC}+\mathrm{CAC}$ & 123,63 & 0,92 & 0,50 & 0,21 \\
\hline $\mathrm{FC}+\mathrm{CZ}$ & 125,84 & 0,91 & 0,34 & 0,38 \\
\hline Ideal $^{* *}$ & $400-500$ & 0,85 & 0,30 & $0,24-0,40$ \\
\hline
\end{tabular}

*CAC: 100\% volume de casca de arroz carbonizada; CZ: 100\% volume de cinza de casca de arroz; FC: 100\% volume de fibra de coco; TP: 100\% de volume de terra do paraíso; TP+CAC: 50\% volume de terra do paraíso + 50\% volume casca de arroz carbonizada; TP+CZ: 50\% volume de terra do paraíso + 50\% volume de cinza de casca de arroz: FC+CAC: $50 \%$ volume de fibra de coco + 50\% volume de casca de arroz carboniza: FC+CZ: 50\% volume de fibra de coco + 50\% volume de cinza de casca de arroz carbonizada.

${ }^{* *}$ Valores ideais citados na literatura para os parâmetros avaliados: DS, Bunt (1973); Pt, VERDONCK \& GABRIELS (1988); EA, PENNINGSFED (1983); AD, De BOOT \& VERDONCK (1972). 
Tabela 3 - Altura da planta (AP) e da inserção da primeira flor (IF), número de folhas (NF), distância dos entrenós (DE), número de flores (NFL), comprimento médio das folhas (CMF), largura média das folhas (LMF), comprimento médio das pétalas (CMP) e largura média das pétalas (LMP). UFSM, Santa Maria, 2004.

\begin{tabular}{|c|c|c|c|c|c|c|c|c|c|}
\hline Tratamentos* & $\mathrm{AP} \mathrm{cm}$ & IF cm & NF (ad.) & $\mathrm{DE} \mathrm{Cm}$ & NFL (ad.) & CMF cm & LMF cm & $\mathrm{CM} \mathrm{cm}$ & $\mathrm{LMP} \mathrm{cm}$ \\
\hline CAC & $35,46 b^{*}$ & $23,21 \mathrm{c}$ & $77,58 \mathrm{a}$ & 0,46 & 5,33a & $6,91 \mathrm{c}$ & $1,04 \mathrm{a}$ & $8,49 a b$ & 3,62 bc \\
\hline $\mathrm{CZ}$ & $40,96 \mathrm{ab}$ & $30,13 \mathrm{ab}$ & $74,50 \mathrm{a}$ & 0,55 & 3,92 a & 7,82 bc & 0,95 a & $8,59 \mathrm{ab}$ & 3,85 abc \\
\hline FC & $37,00 \mathrm{~b}$ & $24,83 \mathrm{c}$ & 79,42 a & 0,46 & $4,17 \mathrm{a}$ & $7,24 \mathrm{c}$ & $0,90 \mathrm{a}$ & $7,91 \mathrm{~b}$ & 3,42 c \\
\hline TP & $44,92 \mathrm{a}$ & $31,86 \mathrm{a}$ & $77,08 \mathrm{a}$ & 0,58 & $5,00 \mathrm{a}$ & 9,84 a & $0,98 \mathrm{a}$ & 9,06 a & 4,13 a \\
\hline $\mathrm{TP}+\mathrm{CAC}$ & $38,96 \mathrm{ab}$ & $24,25 \mathrm{c}$ & 74,17 a & 0,52 & $4,17 \mathrm{a}$ & $8,96 a b$ & $1,00 \mathrm{a}$ & $8,74 \mathrm{a}$ & $4,10 \mathrm{a}$ \\
\hline $\mathrm{TP}+\mathrm{CZ}$ & $40,79 \mathrm{ab}$ & 25,54 bc & 79,92 a & 0,51 & 5,83 a & 7,92 bc & $0,95 \mathrm{a}$ & 8,69 a & 4,01 ab \\
\hline $\mathrm{FC}+\mathrm{CAC}$ & $38,29 \mathrm{ab}$ & $25,50 \mathrm{bc}$ & $74,92 \mathrm{a}$ & 0,51 & $4,50 \mathrm{a}$ & 8,06 bc & 0,99 a & 8,92 a & 3,93 ab \\
\hline $\mathrm{FC}+\mathrm{CZ}$ & $41,21 \mathrm{ab}$ & 26,17 bc & 78,67 a & 0,52 & $4,42 \mathrm{a}$ & 8,11 bc & 0,96 a & 8,89 a & 4,15 a \\
\hline Média & 39,70 & 26,45 & 77,03 & 0,51 & 4,67 & 8,10 & 0,97 & 8,66 & 3,90 \\
\hline CV (\%) & 13,79 & 14,05 & 13,78 & 18.34 & 20,02 & 11,44 & 15,39 & 6,31 & 9,12 \\
\hline
\end{tabular}

*CAC: $100 \%$ volume de casca de arroz carbonizada; CZ: 100\% volume de cinza de casca de arroz; FC: 100\% volume de fibra de coco; TP 100\% de volume de terra do paraíso; TP+CAC: 50\% volume de terra do paraíso + 50\% volume casca de arroz carbonizada; TP+CZ: 50\% volume de terra do paraíso $+50 \%$ volume de cinza de casca de arroz: FC+CAC: $50 \%$ volume de fibra de coco + 50\% volume de casca de arroz carboniza: FC+CZ: 50\% volume de fibra de coco $+50 \%$ volume de cinza de casca de arroz carbonizada.

$9,5 \mathrm{~cm}$, sendo a maior altura obtida com o substrato TP $(44,92 \mathrm{~cm})$ e a menor com a CAC $(35,46 \mathrm{~cm})$. Comparando-se o resultado obtido com a altura característica da cultivar estudada $(35 \mathrm{~cm})$, observa-se que todos os tratamentos resultaram em plantas maiores (INTERNATIONAL FLOWER BULB CENTER, 2004).

Verificou-se que as alturas dependeram mais da distância entrenós, do que do número de folhas (Tabela 3). Como exemplo, o substrato FC produziu altura de planta média de $37 \mathrm{~cm}$, possuiu um dos maiores números de folhas (79 folhas), mas com a menor distância de entrenó $(0,46 \mathrm{~cm})$. O inverso ocorreu com o substrato TP, que não diferiu estatisticamente quanto ao numero de folhas de FC, mas obteve a maior altura de planta $(44,9 \mathrm{~cm})$ e a maior distância entrenós $(0,58 \mathrm{~cm})$. Acredita-se que as diferenças observadas na altura de planta entre os substratos devem-se provavelmente a interações complexas entre o ambiente e a qualidade do substrato, reduzindo-a especialmente nos tratamentos CAC e FC.

Observa-se que a altura de inserção da primeira flor seguiu comportamento semelhante à altura de planta, com valor máximo para TP $(31,86 \mathrm{~cm})$ e mínimo para CAC $(23,21 \mathrm{~cm})$ (Tabela 3$)$. Ao realizar-se a diferença das médias, entre os tratamentos, das alturas de planta e inserção da primeira flor, observou-se que a da primeira inserção até o ápice da planta tem-se $13,25 \mathrm{~cm}$, que corresponde a 33,4\%.

Verificou-se que o número de flores por planta encontra-se dentro dos limites para o tamanho do bulbo (INTERNATIONAL FLOWER BULB
CENTER, 2004), ou seja, entre quatro e seis. Estatisticamente, não houve qualquer influencia dos substratos usados no número de flores por planta, confirmando essa variável é um fator genético associado ao tamanho de bulbo, que só pode ser modificado pelo tamanho do broto no plantio, temperatura de cultivo e radiação solar (NARDI, 2003).

O tamanho das folhas diferiu estatisticamente nos tratamentos analisados somente para o comprimento de folha. Não houve diferença estatística para a largura da folha das plantas. Porém, MELLO (2003), NARDI (2000) e TOLOTTI (2001) avaliando a qualidade de hastes em crisântemo de corte, observaram diferença significativa tanto para o comprimento quanto largura.

Os substratos CAC e FC produziram as folhas mais curtas $(6,91$ e $7,24 \mathrm{~cm}$, respectivamente), enquanto que para TP, observaram-se as folhas mais longas $(9,84 \mathrm{~cm})$, diferindo estatisticamente dos demais substratos, exceto para a mistura TP+CAC. A redução do comprimento foliar causado pelo ambiente CAC e FC, em comparação ao TP, foram, respectivamente, 29,8 e 26,4\%, cujos valores podem ser considerados elevados e perceptíveis numa avaliação visual.

Para a qualidade da flor, avaliada pelo comprimento e largura das pétalas, observou-se mais uma vez o substrato TP apresentou o melhor desempenho para o tamanho da flor. Enquanto que o FC foi aquele que apresentou as menores flores, com uma redução de $12,7 \%$ no comprimento e $17,2 \%$ na largura, em relação ao substrato TP. Pode-se afirmar que há uma forte tendência das plantas de lírio ser de 
inferior qualidade foliar e floral quando cultivados nos materiais CAC e FC, embora somente seja observada diferença estatística para o comprimento médio de folha. Provavelmente, a qualidade inferior das folhes deveu-se a elevada porosidade total dos materiais testados, o que determina em vaso menor capacidade de retenção de água, pela existência de maior disponibilidade de água na parte inferior do que na superfície.

\section{CONCLUSÃO}

Os melhores substratos para a produção de lírio em vaso foram terra do paraíso e a mistura fibra de coco mais cinza de casca de arroz. Estes substratos apresentaram um consumo médio de água de 121,65 e 106,78mL e número total de irrigação, durante o ciclo, de 22 e 21 dias, respectivamente.

\section{REFERÊNCIAS}

BAUMGARTEN, A. Methods of chemical and physical evaluation of substrates for plants. In: ENCONTRO NACIONAL DE SUBSTRATOS PARA PLANTAS, 3., 2002, Campinas: Caracterização, manejo e qualidade de substratos para produção de plantas. Anais... Campinas: IAC, 2002. p.94.

BELLÉ, R.A. Apostila didática de floricultura. Santa Maria: editora ufsm, 2010. 142p.

COUTINHO, M.P. et al. Substrato de cavas de extração de argila enriquecido com subprodutos agroindustriais e urbanos para produção de mudas de sesbânia. Revista Árvore, v.30, n.1, p.147-153. 2006.

DE BOODT, M.; VERDONCK, O. The physical properties of the substrates in horticulture. Acta Horticulturae, v.26, n. 4, p.37-44, 1972.

FARIAS. J.A. Contribuição para a silvicultura de Luehea divaricata Martius et Zuccarini (AÇOITACAVALO). 2006. 70f. Dissertação (Mestrado em Engenharia Florestal) - Curso de Pós-graduação em Engenharia Florestal, Universidade Federal de Santa Maria, RS.

FERMINO, M.H.; BELLÉ, S. Substratos hortícolas. In: PETRY, C. (Org.). Plantas Ornamentais: aspectos para a produção. 2.ed. Passo Fundo: UPF, 2008. p.46-58.

GAULAND, D.C.S.P. Relações hídricas em substratos à base de turfas sob o uso dos condicionadores casca de arroz carbonizada ou queimada. 1997. 107f. Dissertação (Mestrado em Agronomia-Solos) - Universidade Federal do Rio Grande do Sul, Porto Alegre, RS.

INTERNATIONAL FLOWER BULB CENTRE. Manual para elección de variedades de bulbosas de flor. Nd. Hillegon, Holanda; Viçosa: CPT, 2004. 34p.

JASMIM, J.M. et al. Fibra de coco e adubação foliar no crescimento e nutrição de Cryptanthus sinuosus. Horticultura Brasileira, v.24, n.3, p.309-314, 2006.

KÄMPF, A.N. (Coord.). Produção comercial de plantas ornamentais. Guaíba: Agropecuária, 2000. 254p.
MELLO, J.B. Ação de ácido giberélico em dias curtos interrompidos em crisântemos de corte (Dendranthema grandiflora Tzvelev.). 2003. 67f. Dissertação (Mestrado em Agronomia) - Curso de Pós-graduação em Agronomia, Universidade Federal de Santa Maria, RS.

MORENO, J.A. Clima do Rio Grande do Sul. Porto Alegre: Secretaria da Agricultura, 1961. 42p.

NARDI, C. Rendimento e qualidade de crisântemo (Dendranthema grandiflora Tzvelev.) cultivar "Snowdon" em diferentes populações e épocas de plantio. 2000. 73f. Dissertação (Mestrado em Agronomia) - Curso de Pós-graduação em Agronomia, Universidade Federal de Santa Maria, RS.

NARDI, C. Vernalização e Fotoperíodo no Lírio (Lilium longiflorum Thunb.) “Snow Queen”. 2003. 134f. Tese (Doutorado em Agronomia) - Curso de Pós-graduação em Agronomia, Universidade Federal de Santa Maria, RS.

PELIZER, L.H. et al. Utilização de resíduos agroindustriais em processos biotecnológicos como perspectiva de redução do impacto ambiental. Journal Technology Management and Innovation, v.2, n.1, p.118-127. 2007.

PENNINGSFELD, F. Kultursubstrate fur den gartenbau, besonders in Deutschland: ein kritischer Überblick. Plant and Soil, v.75, p.269-281, 1983.

PEREIRA, J.R.D. et al. Consumo de água pela cultura do crisântemo cultivada em ambiente protegido. Engenharia Agrícola, v.25, n.3, p.651-659, 2005.

RODRIGUES, T.M. et al. Desenvolvimento de mudas de bromélia-imperial (Alcantarea imperialis) em diferentes substratos. Ciência e Agrotecnologia, v.28, n.4, p.757-763, 2004.

ROH, M.S.; WILKINS, H.F. The effects of bulb vernalization and shoot photoperiod treatments on growth and flowering of Lilium longiflorum Thunb. Cv. Nellie White. Journal of the American Society for Horticultural Science. v.102, n.3, p.229-235, 1977a.

SANTOS, F.G.B. dos. Substratos para produção de mudas utilizando resíduos agroindustriais. 2006. $103 \mathrm{f}$. Dissertação (Mestrado em Ciência do Solo) - Curso de Pósgraduação em Ciência do solo, Universidade Federal Rural de Pernambuco, RS.

SCHMITZ, J.A.K. et al. Propriedades químicas e físicas de substratos de origem mineral e orgânica para o cultivo de mudas em recipientes. UFRGS, Porto Alegre. Ciência Rural, v.32, n.6, p.937-944, 2002.

SILVEIRA, E.B. et al. Pó de coco como substrato para produção de mudas de tomateiro. Horticultura Brasileira, v.20, n.2, p.211-216, 2002.

TOLOTTI, J.C. Efeito de redutores de crescimento em crisântemo (Dendranthema grandiflora Tzvelev) "Snowdon" cultivado em vaso. 2001. 97f. Dissertação (Mestrado em Agronomia) - Curso de Pós-graduação em Agronomia, Universidade Federal de Santa Maria, RS.

VERDONCK, O.; GABRIELS, R. Substrate requirements for plants. Acta Horticulturae, v.221, p.19-23, 1988. 